\title{
Illness Perceptions and Outcomes in Patients with Inflammatory Bowel Disease: Is Coping a Mediator?
}

\author{
S. J. H. van $\operatorname{Erp}^{1}$ • L. K. M. P. Brakenhoff ${ }^{1}$ M. Vollmann ${ }^{2,3} \cdot$ D. van der Heijde ${ }^{4}$. \\ R. A. Veenendaal $^{1}$ • H. H. Fidder ${ }^{5}$ - D. W. Hommes ${ }^{1,6}$ - A. A. Kaptein ${ }^{7}$. \\ Andrea E. van der Meulen-de Jong ${ }^{1}$ - M. Scharloo ${ }^{7}$
}

Published online: 18 October 2016

(C) International Society of Behavioral Medicine 2016

\begin{abstract}
Purpose Patients with inflammatory bowel disease (IBD) often experience severe impairment in different life domains. Psychological factors, such as illness perceptions and coping, may play a role in the adjustment to IBD as indicated by mental and physical health, activity, and work impairment. The present study aimed at examining the assumption of the Common Sense Model (CSM) that coping mediates the relationship between illness perceptions and adjustment in patients with IBD.

Method In a cross-sectional design, 211 IBD patients (73\% Crohn's disease, $40 \%$ male, mean age $42.9 \pm 12.9$ years) attending an outpatient clinic completed questionnaires assessing illness perceptions (IPQ-R), coping (CORS), mental and physical health (SF-36), as well as activity and work impairment (WPAI). Multiple mediation analyses were applied
\end{abstract}

S. J. H. van Erp and L. K. M. P. Brakenhoff contributed equally to this manuscript.

Andrea E. van der Meulen-de Jong

ae.meulen@lumc.nl

1 Department of Gastroenterology and Hepatology, Leiden University Medical Center, Leiden, the Netherlands

2 Department of Health Psychology, University of Hagen, Hagen, Germany

3 Department of Clinical and Health Psychology, University of Utrecht, Utrecht, the Netherlands

4 Department of Rheumatology, LUMC, Leiden, the Netherlands

5 Department of Gastroenterology and Hepatology, UMCU, Utrecht, the Netherlands

6 Center for Inflammatory Bowel Diseases, UCLA Health System, Los Angeles, CA, USA

7 Department of Medical Psychology, LUMC, Leiden, the Netherlands that allow estimating the total and direct effects of all illness perception dimensions and the indirect effects through all coping strategies on the illness outcomes simultaneously.

Results The analyses yielded significant direct effects of perceptions regarding the cyclical course, the chronic course, the severity of the consequences, the comprehensibility, and the emotional impact of IBD on study outcomes. Additionally, significant indirect effects were found for the perceptions regarding the severity of the consequences, the possibility of personal control, and the comprehensibility of IBD on mental and physical health as well as activity impairment through the use of one specific coping strategy, i.e., reduction of activity. Conclusion The results provide evidence for the assumptions of the CSM and suggest the importance of addressing illness perceptions and activity stimulation in quality health care for IBD patients.

Keywords Inflammatory bowel disease $\cdot$ Common Sense Model $\cdot$ Illness perceptions · Coping · Quality of life · Work productivity

\section{Introduction}

Inflammatory bowel disease (IBD), including Crohn's disease (CD) and ulcerative colitis (UC), is a chronic, relapsing, and remitting disease of the gastrointestinal tract. The clinical course of IBD may be unpredictable and complicated, not only because of intestinal symptoms but also because of the presence of extra-intestinal manifestations. Joint complaints are the most common extra-intestinal manifestations in patients with IBD and cause significant morbidity $[1,2]$. The debilitating symptoms and complications of IBD often affect the patients' physical, mental, and social well-being, resulting in an impaired quality of life (QoL) compared to the general 
population [3]. The diagnosis of IBD can be stressful and often causes psychological problems, such as feelings of hostility, despair, denial, sadness, grief, and anxiety [4, 5], which can induce long-term psychosocial impairments and negatively affect overall QoL [6, 7]. Furthermore, the uncertainty of the disease course makes it challenging for patients to make decisions regarding their work and daily activities, which may result in unemployment or social isolation $[6,8,9]$. Although work disability has been generally related to the severity of IBD, the contribution of psychological factors remains unclear [10].

The Common Sense Model (CSM) [11] of self-regulation of health and illness is a useful framework to understand how psychological factors, i.e., illness perceptions and coping, influence illness outcomes in IBD patients [3, 12-17]. The CSM proposes that patients develop their own ideas about their illness, i.e., illness perceptions, in order to understand the health threat. Illness perceptions embrace five core dimensions, namely (1) ideas about the identity of the illness consisting of the label as well as the symptoms that are associated with the illness, (2) ideas about the causes of the illness, (3) ideas about the consequences that the illness create, (4) ideas about to what extent the illness can be controlled or cured by own behavior or medical treatment, and (5) ideas about the course of the illness and the duration of the symptoms [11]. Two additional dimensions refer to (6) the emotional impact caused by the illness and (7) the overall comprehensibility of the illness [18]. The CSM proposes further that illness representations determine how patients will cope with their illness, and therefore which cognitive and behavioral strategies they will use in order to deal with their illness. Many different coping strategies depending on the illness have been identified, such as turning to religion, ignoring the illness, seeking social support, withdrawal from activities, venting emotions, wishful thinking, distraction, acceptance, and positive reinterpretation $[19,20]$. According to the CSM, the used coping strategies have an effect on the adjustment to an illness as indicated, for example, by psychological, physical, and social well-being. In summary, the CSM maintains that illness perceptions influence adjustment to the illness by triggering the use of certain coping strategies.

Studies using the CSM in patients with IBD have shown that illness perceptions significantly accounted for 11 to $21 \%$ of the variance in QoL [3, 12, 21-23]. Patients who believed their illness would last a short time displayed high QoL scores, while patients who believed their IBD resulted in severe consequences were more likely to have lower QoL scores, thus indicating the contradictory effect of different illness perceptions on patients' QoL [21]. Van der Have et al. [22, 23] found associations between illness perceptions and work disability and activity impairment in IBD patients with joint and/or back pain. Patients who believe in serious consequences and with a perception of weak personal control avoid situations in which they could experience limitations, resulting in more work disability and more activity impairment [22-24].

Also, previous research showed that illness perceptions influence coping behavior of IBD patients. Patients with IBD, who perceive their illness as understandable and controllable, display more active coping (e.g., problem-focused coping) [12].

Furthermore, coping has been found to be associated with QoL, daily activity, and work impairment in patients with IBD $[22,25]$. In a cross-sectional study, Parekh et al. [5] demonstrated that confrontational, evasive, and optimistic coping strategies are the most widely adopted and the most effective ones among patients with IBD [5]. QoL was significantly higher for those patients who primarily used these adaptive coping strategies compared to patients who used maladaptive coping strategies including substance use, behavioral disengagement, self-blame, denial, venting emotions, and selfdistraction [5, 25]. In addition, van der Have et al. [22, 23] showed that coping behavior influences illness outcomes, with the maladaptive coping strategy "reduction of physical activity" being significantly associated with a reduced QoL and more daily activity impairment [22].

\section{The Present Study}

The purpose of the present study was to investigate the assumption of the CSM that coping mediates the relationship between illness perceptions and adjustment. Adjustment is conceptualized here as mental and physical health as well as activity and work impairment in patients with IBD. Complex multiple mediation analyses that allow estimating the total and direct associations of all illness perception dimensions and the indirect associations through all coping strategies on adjustment simultaneously were used. Based on previous research, we hypothesized that negative illness perceptions (e.g., severe consequences and strong emotional representations) would be associated with a reduced mental and physical health as well as more activity and work impairment. Additionally, we expected to find that coping mediates the effects of illness perceptions on the outcomes. Compared to other studies that have investigated the mediation hypothesis of the CSM with separate mediation analyses for each illness perception dimension and/or coping strategy in patients with IBD [22], the present analyses better reflect the proposed theoretical model.

\section{Methods}

\section{Patient Population and Data Collection}

Between September 2009 and February 2010, 255 IBD patients attending the IBD outpatient clinic of the Leiden University Medical Center (LUMC), the Netherlands, were 
requested to take part in our study. No exclusion or inclusion criteria were specified. The participants had to complete webbased or postal questionnaires assessing demographic characteristics, disease activity, peripheral joint pain, back pain, illness perceptions about IBD, coping strategies, mental and physical health, as well as activity and work impairment $[22$, 26]. Of the 255 patients, 245 completed all questionnaires. Finally, the data of the 211 patients who completed the Revised Illness Perception Questionnaire (IPQ-R) regarding their IBD complaints were analyzed. The remaining 34 IBD patients who completed the IPQ-R regarding other diseases, were excluded. The study was approved by the medical ethical committee of the LUMC and all patients signed informed consent.

\section{Measures}

Disease Activity In patients with Crohn's disease, the HarveyBradshaw Index (HBI) was used to measure clinical disease activity [27]. The HBI used for the present study consisted of in total 11 items, including general well-being, abdominal pain, daily number of liquid stools, and extra-intestinal manifestations (arthralgia, uveitis, erythema nodosum, apthous ulcers, pyoderma gangrenosum, anal fissure, new fistula, and abscess). This web-based questionnaire was completed at home and therefore the question about abdominal mass was excluded.

In patients with ulcerative colitis, the disease activity was measured by using the Simple Clinical Colitis Activity Index (SCCAI) [28] consisting of 9 items: bowel frequency (during the day), bowel frequency (at night), urgency of defecation, blood in stool, general well-being and extra-colonic features (arthritis, uveitis, pyoderma gangrenosum, and erythema nodosum). In both the HBI and SCCAI, a score above 4 reflects active disease.

Peripheral Joint and/or Back Pain At study inclusion, participants were asked (1) whether they currently have painful and/or swollen peripheral joints and (2) whether they currently have back pain that already lasts for 3 months or longer. If one of these questions was answered with yes, it was coded that peripheral joint pain and/or back pain was present [26].

Illness Perceptions Illness perceptions about IBD were assessed with the revised version of the IPQ-R, a widelyused questionnaire that has been validated in different patient samples [18]. The IPQ-R contains three parts.

The first part measures illness identity and includes 15 common symptoms that patients can attribute to their IBD (yes $=1$ or no $=0$ ), with a summary score ranging from 0 to 15. A higher score represents a stronger belief that the symptoms are part of the IBD.
The second part contains 7 subscales measuring chronic timeline (perceived duration of IBD, 6 items), cyclical timeline (perceived variability in the symptoms of IBD, 3 items), consequences (perceived impact of IBD on patients' life, 6 items), personal control (perceived effectiveness of controlling IBD by own behavior, 6 items), treatment control (perceived effectiveness of controlling IBD by treatment, 5 items), coherence (extent of understanding IBD, 4 items), and emotional representations (perceived emotional impact of IBD, 6 items). Items were answered on a 5-point Likert scale ranging from 1 "strongly disagree" to 5 "strongly agree." For each subscale, mean scores were computed after recoding inversely formulated items. Higher scores reflect stronger beliefs on that particular subscale. Internal reliability of the subscales was relatively high with Cronbach's alphas ranging from .78 (cyclical timeline) to .88 (emotional representations). For treatment control, however, Cronbach's alpha was .59, indicating that in this patient group beliefs about controlling IBD, improving IBD, and curing IBD do not form a homogeneous scale.

The third part, questions about causal attributions, used the same 5-point scale and consisted of 17 items representing perceived causes of IBD. As the underlying dimensions of perceived causes may vary between diseases, Moss-Morris et al. suggested to perform a principal component analysis (PCA) with VARIMAX-rotation on the causal items [18]. The PCA of causal items produced 4 factors accounting for $59 \%$ of the total variance. Items with a factor loading higher than .50 were interpreted to represent a particular factor. The first factor accounted for $31 \%$ of the variance and included 10 items (stress, own behavior, mental attitude, family problems, working too hard, emotional state, getting older, alcohol use, smoking, and personality) and was labelled stress and (stress) behavior. Cronbach's alpha of this factor was .90. The second (3 items: virus, diet, bad luck) and third ( 3 items: poor medical treatment, environment, injury) factors both accounted for $10 \%$ of the variance but demonstrated insufficient internal reliability (Cronbach's alphas <.50) and were therefore excluded from further analyses. The fourth factor accounted for $7 \%$ of the variance, consisted of only one item (heredity) and was also excluded from further analyses. The items of the first factor were averaged and a higher score indicates stronger beliefs in stress and (stress) behavior causing the illness.

Coping Strategies Coping strategies were assessed with the Coping with Rheumatic Stressors Questionnaire [19]. This questionnaire was used because most of the IBD patients in this cohort were diagnosed with joint complaints. The CORS measures eight strategies of coping with pain, with limitations and with dependence, which are the most important stressors in inflammatory rheumatic diseases. Coping strategies related to pain included comforting cognitions (i.e., positive self-instructions, 9 items), decreasing activity (i.e., reduce activity and take more rests, 8 items), and diverting attention (doing or 
thinking of nice things instead of focusing on the pain, 8 items). Three scales refer to limitations, namely optimism (i.e., positive thinking, 5 items), pacing (i.e., lowering the number and intensity of activities, 10 items), and creative solution seeking (i.e., thinking of new ways to get things done, 8 items). Coping styles related to dependence included acceptation (i.e., take the dependence for granted) and consideration (i.e., trying to be useful to others). Items were answered on a 4-point scale from 1 "seldom or never" to 4 "very often." Mean scores were calculated with higher scores indicating more frequent use of that particular coping strategy. Internal reliability within the subscales was high (Cronbach's alpha ranging from .79 to .85 ).

Quality of Life The SF-36 is a generic questionnaire that measures eight general health concepts, which are grouped within a mental (MCS) and a physical (PCS) component summary score that represent mental health and physical health [29]. MCS consists of the concept vitality, social function, role limitations due to personal or emotional problems, and mental health. Physical functioning, role limitations due to physical health problems, bodily pain, and general health perception are included in the PCS. The score for subdomains range from 0 to 100 , with higher scores representing better mental and physical health, respectively [29].

Activity and Work Impairment Two items of the Work Productivity and Activity Impairment Questionnaire (WPAI) were used to measure activity impairment and work impairment during the past 7 days [30]. The WPAI has been validated in a number of diseases including IBD [31]. Patients were asked to indicate on 11-point scale ranging from 0 "not at all" to 10 "completely" how much their IBD affected (1) their ability to do their regular daily activities and (2) their productivity while working. The last question was only answered by employed patients. Scores were multiplied with 100 in order to compute percentages from 0 to 100 , with a higher percentage indicating greater impairment.

\section{Statistical Analysis}

All statistical analyses were performed with IBM SPSS Statistics Version 22.

In a first step, bivariate associations between the study variables were analyzed by means of Pearson correlation analyses. This procedure allows comparing our results with findings of previous studies that reported Pearson correlation coefficients by default.

In a second step, four parallel multiple mediation analyses [32] (one for each illness outcome) were calculated with the illness perception dimensions as independent variables, the coping strategies as mediators, and the illness outcome as dependent variable. In order to keep the number of variables to a minimum, only those illness perception dimensions and coping strategies that were significantly correlated with the illness outcome were included in the respective mediation analysis. As previous research has shown that disease activity [25] and the experience of pain [22] has a strong impact on illness outcomes in patients with IBD, disease activity, as well as present back pain and/or peripheral joint pain were entered as control variables in all analyses.

The mediation analyses were performed by using the MEDIATE-macro for SPSS [33] that consecutively runs a number of analyses that estimate the total, direct, and indirect effects of illness perceptions on the respective illness outcome. The total and direct effects were estimated by means of a stepwise multiple regression analysis in which illness perception dimensions were entered in the first step and coping strategies were entered in the second step. Total effects refer to the specific relationships between each illness perception dimension and the respective illness outcome while controlling for the effect of all other illness perceptions dimensions (first step), and direct effects refer to the specific relationships between each illness perception dimension and the respective illness outcome while controlling for all other illness perception dimensions and all coping strategies (second step). The effects of illness perceptions on coping were estimated by calculating multiple regression analyses with all illness perception dimensions as predictors and the respective coping strategy as outcome. The specific indirect effects of the illness perception dimensions on the respective illness outcome through each coping strategy and their significance were determined by means of the new standard method of estimating indirect effects [32], i.e., bootstrap analyses with 5000 bootstrap samples. As recommended by Hayes [32], coefficients will be reported in unstandardized form in order to correctly interpret bootstrap confidence intervals and to map the results directly onto the measurement scales.

\section{Results}

\section{Sample}

The sample consisted of 211 IBD patients with a mean age of 42.9 years $(\mathrm{SD}=12.9)$ and a mean disease duration of 15.9 years $(\mathrm{SD}=11.3)$. The majority of the participants was diagnosed with $\mathrm{CD}$, was female, and had axial and/or peripheral joint complaints. Clinical and demographic characteristics of the study population are shown in Table 1 .

\section{Bivariate Associations Between Illness Representations, Coping Strategies, and Illness Outcomes}

The results of the correlation analyses are presented in Table 2 . Nearly all illness perception dimensions were significantly 
Table 1 Clinical and demographic characteristics of the study population $(n=211)$

\begin{tabular}{|c|c|}
\hline \multicolumn{2}{|l|}{ Type of IBD, n (\%) } \\
\hline Crohn's disease & $154(73.0)$ \\
\hline Ulcerative colitis & $57(27.0)$ \\
\hline Age (years), mean $\pm \mathrm{SD}$ & $42.9 \pm 12.9$ \\
\hline Male, $n(\%)$ & $84(39.8)$ \\
\hline Disease duration (years), mean $\pm \mathrm{SD}$ & $15.9 \pm 11.3$ \\
\hline Employed, $n(\%)$ & $137(64.9)$ \\
\hline \multicolumn{2}{|l|}{ Montreal classification: } \\
\hline Location $\mathrm{CD}, n(\%)$ & $n=154$ \\
\hline L1 ileal & $38(24.7)$ \\
\hline L2 colonic & $30(19.5)$ \\
\hline L3 ileocolonic & $72(46.8)$ \\
\hline L4 upper & $1(0.7)$ \\
\hline $\mathrm{L} 1-3+\mathrm{L} 4$ & $13(8.4)$ \\
\hline \multicolumn{2}{|l|}{ Behavior $\mathrm{CD}, n(\%)$} \\
\hline B1 non-stricturing/penetrating & $67(43.5)$ \\
\hline B2 stricturing & $22(14.3)$ \\
\hline B3 penetrating & $21(13.6)$ \\
\hline+ perianal disease & $44(28.6)$ \\
\hline Extension UC, $n(\%)$ & $n=57$ \\
\hline E1 ulcerative proctitis & $5(8.8)$ \\
\hline E2 left sided UC & $17(29.8)$ \\
\hline E3 extensive UC (pancolitis) & $35(61.4)$ \\
\hline \multicolumn{2}{|l|}{ Medication, $n(\%)$} \\
\hline Mesalazine & $55(26.1)$ \\
\hline Corticosteroids & $26(12.3)$ \\
\hline Immunosuppressive drugs (Aza/6MP/MTX) & $75(35.5)$ \\
\hline Anti-TNF & $74(35.1)$ \\
\hline None & $47(22.3)$ \\
\hline \multicolumn{2}{|l|}{ Control variables } \\
\hline Joint and/or back pain & $124(58.8)$ \\
\hline Active IBD disease & $83(39.3)$ \\
\hline
\end{tabular}

correlated with outcome measures. More specifically, a strong illness identity, perceptions of a cyclical timeline, of serious consequences, of low personal, and of low treatment control as well as low coherence, strong emotional representations, and strong stress and (stress) behavior attributions were associated with lower levels of mental and physical health as well as elevated levels of impairment.

Various coping strategies also showed significant correlations with illness outcomes. More specifically, more frequent use of the coping strategies decreasing activities to cope with pain and pacing to cope with limitations were related to lower physical and mental health and to more activity and work impairment. In addition, lower optimism was related to worse mental health, while the more frequent use of creative solution seeking and showing consideration was related to worse physical health.
Also, various significant correlations were observed between illness perception dimensions and coping strategies. For example, a strong illness identity, more cyclical timeline perceptions, the perception of serious consequences, and low illness coherence were associated with more frequent use of both coping strategies decreasing activity and pacing. Furthermore, the perception of less serious consequences, less strong emotional representations, and the perception of high personal and treatment control were related to more frequent use of optimism. Also, strong illness identity, acute timeline perceptions, the perception of serious consequences, and the perception of high personal control were associated with more creative solution seeking. Finally, more cyclical timeline perceptions and perceptions of high personal control were correlated with more frequent use of showing consideration.

\section{Total, Direct, and Indirect Effects of Illness Perceptions on Illness Outcomes (Through Coping Strategies)}

Mental Health The mediation analysis with mental health as dependent variable included the illness representation dimensions identity, timeline cyclical, consequences, personal control, treatment control, illness coherence, emotional representations, and stress and (stress) behavior attributions as independent variable. The coping strategies decreasing activity, optimism, and pacing as mediators, as well as disease activity and present back and/or peripheral joint pain were included as control variables. The results of the analysis are displayed in Table 3 and Fig. 1a. The regression analysis revealed significant total and direct effects for illness coherence and emotional representations on mental health, indicating that low illness coherence and strong emotional representations were associated with worse mental health. Furthermore, bootstrap analyses revealed significant indirect effects of consequences, -0.63 , BCa $95 \%$ CI $(-1.495,-0.072)$, personal control, -0.34 , BCa $95 \%$ CI $(-0.999,-0.006)$, and illness coherence, $0.39, \mathrm{BCa} 95 \% \mathrm{CI}(0.026,1.043)$ on mental health through decreasing activity. Stronger perceptions of serious consequences and of personal control as well as low illness coherence were associated with the more frequent use of decreasing activity, which in turn was related to lower levels of mental health. All variables together explained $35 \%$ of the variance in mental health.

Physical Health The mediation analysis with physical health as dependent variable included the illness representation dimensions identity, timeline cyclical, consequences, personal control, treatment control, illness coherence, and emotional representations as independent variables. The coping strategies decreasing activity, pacing, seeking solutions, and showing consideration as mediators, as well as disease activity and present back and/or peripheral joint pain were included as control variables. The results of the analysis are displayed in 
Table 2 Means and standard deviations of the study variables and intercorrelations between illness perceptions [1 to 9], coping [10 to 17], and illness outcomes

\begin{tabular}{|c|c|c|c|c|c|c|c|c|c|c|c|c|c|c|}
\hline & 10 & 11 & 12 & 13 & 14 & 15 & 16 & 17 & $\begin{array}{l}\text { Mental } \\
\text { health }\end{array}$ & $\begin{array}{l}\text { Physical } \\
\text { health }\end{array}$ & $\begin{array}{l}\text { Activity } \\
\text { impairment }\end{array}$ & $\begin{array}{l}\text { Work } \\
\text { impairment }\end{array}$ & $M$ & SD \\
\hline 1. Identity & .01 & $.30 * * *$ & .13 & -.08 & $.32 * * *$ & $.20 * *$ & .05 & .07 & $-.25^{* * * *}$ & $-.36^{* * *}$ & $.34 * * *$ & $.22 * *$ & 4.74 & 2.84 \\
\hline 2. Timeline chronic & -.03 & -.05 & $-.20 * *$ & -.08 & .02 & $-.14^{*}$ & .03 & .01 & -.10 & .04 & -.02 & $-.18^{*}$ & 4.30 & 0.58 \\
\hline 3. Timeline cyclical & $.18 * *$ & $.24 * * *$ & .12 & .02 & $.15^{*}$ & .10 & .05 & $.18 *$ & $-.18 * *$ & $-.34 * * *$ & $.31 * * *$ & $.29 * * *$ & 3.60 & 0.82 \\
\hline 4. Consequences & -.04 & $.41 * * *$ & .003 & $-.15^{*}$ & $.37 * * *$ & $.14 *$ & .02 & .04 & $-.41 * * *$ & $-.48 * * *$ & $.49 * * *$ & $.39 * * *$ & 3.06 & 0.83 \\
\hline 5. Personal control & .11 & -.01 & $.17 *$ & $.22 * *$ & -.003 & $.21 * *$ & $.21 * *$ & $.18^{* * *}$ & $.21 * *$ & $.23 * *$ & $-.19 * *$ & -.09 & 2.93 & 0.69 \\
\hline 6. Treatment control & .07 & $-.17 *$ & .13 & $.18 * *$ & -.12 & .07 & -.01 & -.03 & $.17 *$ & $.28 * * *$ & $-.29 * * *$ & $-.18 *$ & 3.19 & 0.55 \\
\hline 7. Illness coherence & .03 & $-.23 * *$ & .02 & .05 & $-.15^{*}$ & -.08 & .01 & -.08 & $.44 * * *$ & $.19 * *$ & $-.26^{* * *}$ & $-.22 *$ & 3.73 & 0.76 \\
\hline $\begin{array}{l}\text { 8. Emotional } \\
\text { representations }\end{array}$ & $-.18^{*}$ & $.21 * *$ & -.11 & $-.29 * * *$ & .11 & .04 & -.10 & .003 & $-.54 * * *$ & $-.22 * *$ & $.32 * * *$ & $.24 * *$ & 2.61 & 0.83 \\
\hline $\begin{array}{l}\text { 9. Stress and behavior } \\
\text { attributions }\end{array}$ & -.04 & .11 & .10 & -.04 & .07 & .08 & .00 & .05 & $-.20 * *$ & -.07 & $.16^{*}$ & $.21 * *$ & 2.24 & 0.75 \\
\hline $\begin{array}{l}\text { 10. Comforting } \\
\text { cognitions }\end{array}$ & - & - & - & - & - & - & - & - & .01 & -.13 & .02 & .06 & - & - \\
\hline 11. Decreasing activity & - & - & - & - & - & - & - & - & $-.29 * * *$ & $-.39 * * *$ & $.41 * * *$ & $.30 * * *$ & - & - \\
\hline 12. Diverting attention & - & - & - & - & - & - & - & - & -.03 & -.12 & .01 & .06 & - & - \\
\hline 13. Optimism & - & - & - & - & - & - & - & - & $.16^{*}$ & .03 & -.08 & -.09 & - & - \\
\hline 14. Pacing & - & - & - & - & - & - & - & - & $-.16^{*}$ & $-.38 * * *$ & $.34 * * *$ & $.19 * *$ & - & - \\
\hline 15. Solution seeking & - & - & - & - & - & - & - & - & -.10 & $-.17 *$ & .10 & .09 & - & - \\
\hline $\begin{array}{l}\text { 16. Accepting } \\
\text { dependence }\end{array}$ & - & - & - & - & - & - & - & - & .07 & .000 & -.01 & -.04 & - & - \\
\hline $\begin{array}{l}\text { 17. Showing } \\
\text { consideration }\end{array}$ & - & - & - & - & - & - & - & - & -.10 & $-.16^{*}$ & .05 & .08 & - & - \\
\hline$M$ & 2.89 & 2.24 & 2.36 & 2.93 & 2.33 & 2.48 & 2.13 & 2.70 & 46.74 & 46.99 & 36.07 & 23.14 & - & - \\
\hline SD & 0.55 & 0.53 & 0.57 & 0.60 & 0.56 & 0.54 & 0.62 & 0.52 & 9.75 & 9.56 & 29.26 & 29.02 & - & - \\
\hline
\end{tabular}

$* * * p<.001, * * p<.01, * p<.05$

Table 3 and Fig. 1b. The multiple regression analysis revealed significant total and direct effects of consequences, disease activity, and present back and/or peripheral joint pain on physical health, indicating that stronger perceptions of serious

Table 3 Total and direct effects of illness perception dimensions and direct effects of coping strategies on illness outcomes

\begin{tabular}{|c|c|c|c|c|c|c|c|c|}
\hline & \multicolumn{2}{|c|}{ Mental health } & \multicolumn{2}{|c|}{ Physical health } & \multicolumn{2}{|c|}{ Activity impairment } & \multicolumn{2}{|c|}{ Work impairment } \\
\hline & B step 1 & B step 2 & B step 1 & B step 2 & B step 1 & B step 2 & B step 1 & B step 2 \\
\hline Step 1: & \multicolumn{2}{|c|}{$\begin{aligned} \text { adj. } R^{2} & =.34, F(10, \\
200) & =11.93^{* * *}\end{aligned}$} & \multicolumn{2}{|c|}{$\begin{aligned} \text { adj. } R^{2} & =.39, F(9, \\
201) & =15.99^{* * *}\end{aligned}$} & \multicolumn{2}{|c|}{$\begin{aligned} \text { adj. } R^{2} & =.35, F(10, \\
200) & =12.50^{* * *}\end{aligned}$} & \multicolumn{2}{|c|}{$\begin{array}{c}\text { adj. } R^{2}=.25, F(10, \\
126)=5.59^{* * *}\end{array}$} \\
\hline Identity & -0.12 & -0.09 & -0.31 & -0.21 & 0.76 & 0.47 & 1.11 & 0.85 \\
\hline Timeline chronic & - & - & - & - & - & - & $-17.23 * *$ & $-16.45^{* *}$ \\
\hline Timeline cyclical & 0.70 & 0.88 & $-1.77 *$ & -1.38 & 3.30 & 2.46 & 2.93 & 2.60 \\
\hline Consequences & -1.37 & -0.97 & $-3.16^{* *}$ & $-2.24 *$ & $9.29 * *$ & $6.50 *$ & $9.43 * *$ & $8.81 *$ \\
\hline Personal control & 1.08 & 1.30 & 0.77 & 1.35 & -0.49 & -1.90 & - & - \\
\hline Treatment control & 0.00 & -0.22 & 1.12 & 0.75 & -5.05 & -4.26 & $-10.57^{*}$ & $-10.38 *$ \\
\hline Illness coherence & $2.23 *$ & $1.97 *$ & 0.84 & 0.25 & -3.33 & -1.70 & 0.71 & 0.65 \\
\hline Emotional representations & $-4.01 * * *$ & $-4.08 * * *$ & 1.51 & 0.98 & -0.23 & 0.97 & 1.23 & 1.09 \\
\hline Stress and (stress) behavior attributions & -0.51 & -0.52 & - & - & 2.08 & 2.25 & 1.31 & 0.99 \\
\hline Control variable: active IBD & -2.10 & -1.99 & $-2.54 * *$ & $-2.46^{* *}$ & 6.03 & 5.67 & 5.90 & 5.66 \\
\hline Control variable: pain & -0.98 & -1.07 & $-5.78 * * *$ & $-5.46^{* * *}$ & $15.04 * * *$ & $14.74 * * *$ & 5.15 & 5.29 \\
\hline Step 2: & \multicolumn{2}{|c|}{$\begin{array}{c}\text { adj. } R^{2}=.35, F(13 \\
197)=9.62^{* * *}\end{array}$} & \multicolumn{2}{|c|}{$\begin{aligned} \text { adj. } R^{2} & =.42, F(13 \\
197) & =12.60^{* * *}\end{aligned}$} & \multicolumn{2}{|c|}{$\begin{aligned} \text { adj. } R^{2} & =.38, F(12 \\
198) & =11.91^{* * *}\end{aligned}$} & \multicolumn{2}{|c|}{$\begin{array}{c}\text { adj. } R^{2}=.25, F(12 \\
124)=4.79^{* * *}\end{array}$} \\
\hline Decreasing activity & - & $-2.89^{*}$ & - & -2.35 & - & $10.09^{*}$ & - & 6.62 \\
\hline Optimism & - & 0.17 & - & - & - & - & - & - \\
\hline Pacing & - & 0.95 & - & -2.01 & - & 2.20 & - & -1.24 \\
\hline Solution seeking & - & - & - & 0.67 & - & - & - & - \\
\hline Showing consideration & - & - & - & -1.38 & - & - & - & - \\
\hline
\end{tabular}

An empty cell means that the respective illness perception dimension/coping strategy was not included in the analysis. Unstandardized coefficients are reported. B's of step 1 represent total effects and B's of step 2 represent direct effects

$* * * p<.001, * * p<.01, * p<.05$ 
a

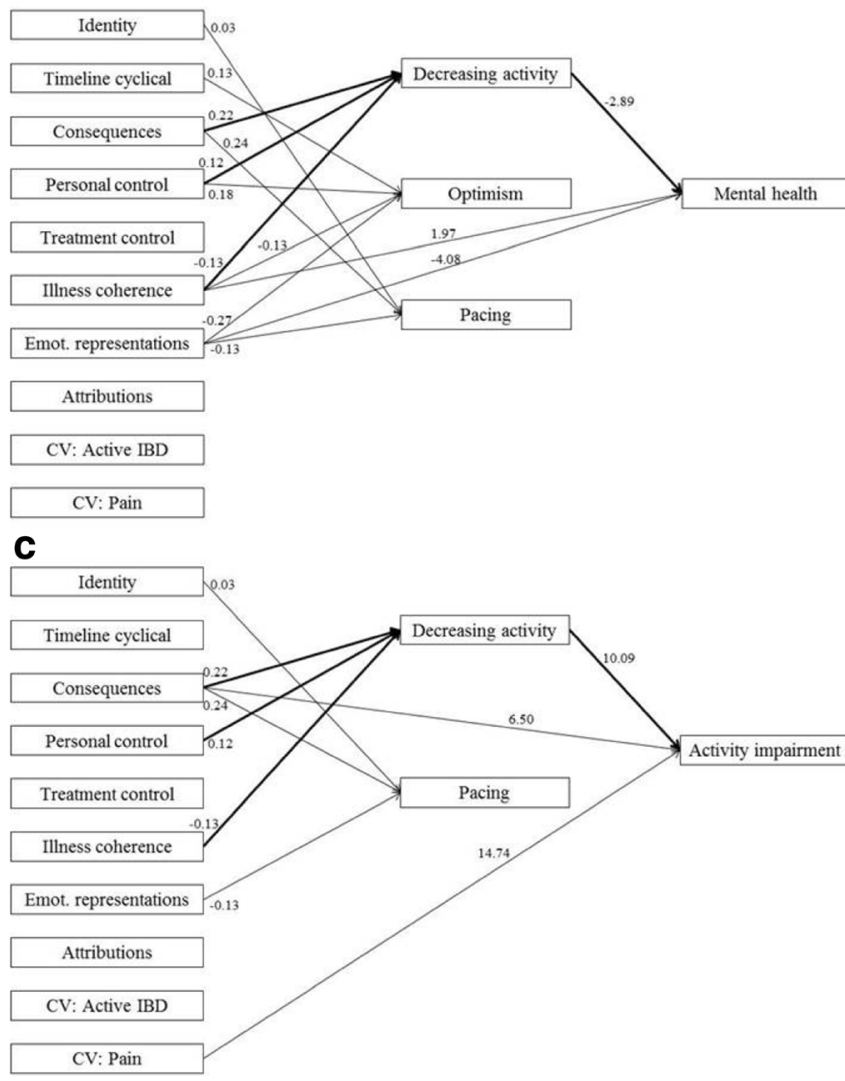

b

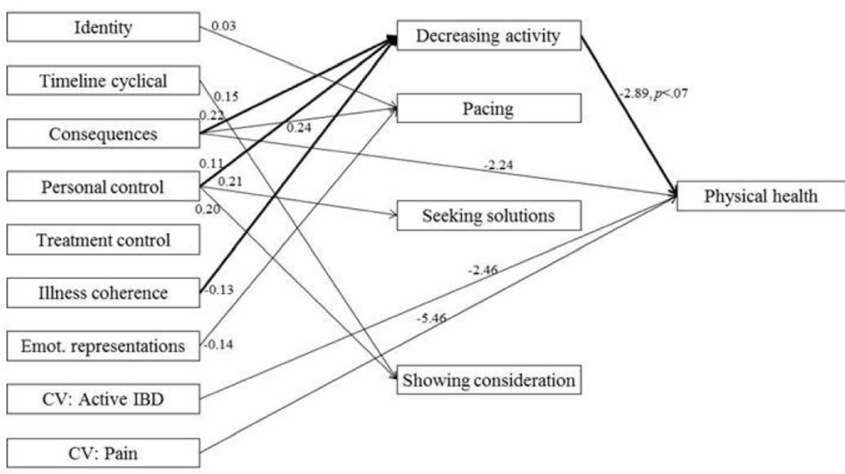

d

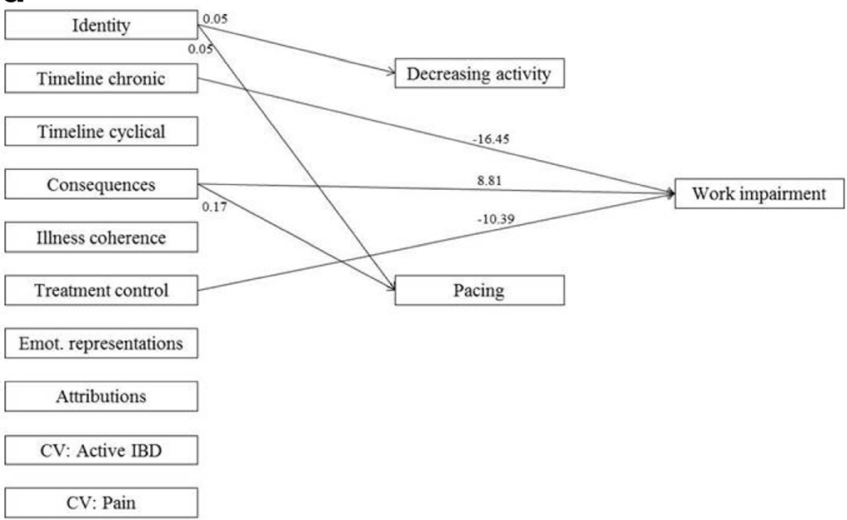

Fig. 1 Results of the four mediation analyses. Only paths that are significant at $p<.05$ are displayed, unless stated otherwise. Unstandardized coefficients are reported. Significant indirect effects are indicated by bold printed paths. $C V$ control variable

consequences and more disease activity and pain were associated with lower levels of physical health. Furthermore, bootstrap analyses again revealed significant indirect effects of consequences, -0.51 , BCa $95 \%$ CI $(-1.297,-0.074)$, personal control, -0.27 , BCa $95 \% \mathrm{CI}(-0.842,-0.011)$, and illness coherence, 0.31 , BCa $95 \%$ CI $(0.014,1.015)$ on physical health through decreasing activity. Stronger perceptions of serious consequences and of personal control as well as low illness coherence were associated with a more frequent use of decreasing activity, which in turn was related to worse physical health. All variables together explained $42 \%$ of the variance in physical health.

Activity Impairment The mediation analysis with activity impairment as dependent variable included the illness representation dimensions identity, timeline cyclical, consequences, personal control, treatment control, illness coherence, emotional representations, and stress and (stress) behavior attributions as independent variables. The coping strategies decreasing activity and pacing as mediators, as well as disease activity and present back and/or peripheral joint pain were included as control variables. The results of the analysis are displayed in Table 3 and Fig. 1c. The multiple regression analysis revealed significant total and direct effects of consequences and present back and/or peripheral joint pain on activity impairment, indicating that strong perceptions of negative consequences and more pain were related to more activity impairment. Furthermore, bootstrap analyses again revealed significant indirect effects of consequences, 2.20 , BCa $95 \% \mathrm{CI}(0.633,4.890)$, personal control, $1.19, \mathrm{BCa} 95 \% \mathrm{CI}(0.156,3.020)$, and illness coherence, -1.36 , BCa $95 \%$ CI $(-3.55,-0.241)$ on activity impairment through decreasing activity. Stronger perceptions of serious consequences and personal control as well as low illness coherence were related to the more frequent use of decreasing activity, which in turn was related to higher levels of activity impairment. All variables together explained $38 \%$ of the variance in activity impairment.

Work Impairment The mediation analysis with work impairment as dependent variable included the illness representation dimensions identity, timeline chronic, timeline cyclical, consequences, treatment control, illness coherence, emotional representations, and stress and (stress) behavior attributions as independent variables. The coping strategies decreasing activity and pacing as mediators as well as disease activity and present back and/or peripheral joint pain were included as control variables. The results of the analysis are displayed in Table 3 and Fig. 1d. The multiple regression analysis 
revealed significant total and direct effects of timeline chronic, consequences, and treatment control on work impairment, indicating that more acute timeline perceptions, stronger perceptions of serious consequences and weaker perceptions treatment effectiveness were associated with more work impairment. Bootstrap analyses revealed no significant indirect effects. All variables together explained $25 \%$ of the variance in work impairment.

\section{Discussion}

The purpose of this cross-sectional study was to examine the assumption of the CSM [11] that coping mediates the relationship between illness perceptions and adjustment in patients with IBD. To our knowledge, this study was the first to apply multiple mediation analyses that allow estimating the total and direct effects of all illness perception dimensions and the indirect effects through all coping strategies on illness outcomes simultaneously.

The findings of the present study indicate that both illness perceptions and coping play a significant role in adjustment to IBD even after controlling for disease activity and peripheral joint and/or back pain. All variables together could explain a meaningful proportion of the variance in the illness outcomes.

In accordance with the CSM [11] and our hypotheses, the effects of illness perceptions on health outcomes were partially mediated by coping. More specifically, perceptions of more serious consequences, stronger personal control, and a lack of personal understanding of IBD were associated with a more frequent use of the coping strategy decreasing activity, which, in turn, was associated with lower mental health, lower physical health, and more activity impairment. Two aspects of these results are noteworthy: First, within the mediation models decreasing activity was the only coping strategy that showed significant associations with the illness outcomes and served as a mediator between illness perceptions and illness outcomes. Thus, decreasing activity is of special importance in coping with IBD. Earlier studies [12, 13, 22] already provided initial indications on the significance of this coping strategy; however, in our study, the pattern is most pronounced. Secondly, although the bivariate correlations between personal control and illness outcomes suggest a positive impact, the indirect effect of personal control on mental and physical health as well as activity impairment was negative. In general, in the CSM stronger perceptions of personal control are seen as beneficial for adaptation [17, 34], but from our results, it seems that feelings of personal control are expressed in the maladaptive coping strategy decreasing activities. This contradictory finding should be investigated in future research.

Beside the indirect effect via decreasing activity, several illness perceptions dimensions were also directly, i.e., independently from coping, associated with mental health, physical health, and activity impairment. The direction of these associations is in line with the assumptions of the CSM $[11,20]$ and our hypothesis based on previous findings [12-14, 16, 17]. Particularly, a lack of understanding IBD and the experience of more negative emotions are related to worse mental health. Perceptions of more severe consequences have an unfavorable effect on physical health and the capability to engage in daily activity.

Interestingly, with regard to work impairment, only direct effects of illness representation were found. Perceiving IBD as acute, as causing severe consequences, and as being noncontrollable by treatment were linked to more work impairment. The absence of the mediation effect of the coping strategy decreasing activity might be due to the smaller sample size as only employed patients were included.

\section{Limitations}

Some limitations need to be acknowledged. First, the study had a cross-sectional design and therefore, the direction of causality in the associations between illness perceptions, coping, and outcomes could not be determined. Longitudinal studies are needed to address this issue, since these could examine the dynamic processes of illness perceptions and coping on illness outcomes specified in the CSM [11]. Second, the present findings are exclusively based on selfreported measures that might be subject to several forms of bias. Further studies could profit from including objective measures (i.e., medical assessments). Third, although back and/or joint pain appears often in IBD patients, the CORS questionnaire has not been validated in these patients and coping strategies linked to IBD-specific stressors might have been missed. However, this questionnaire has been validated in patients with rheumatoid arthritis [18], an inflammatory joint disease. Future studies investigating the influence of coping on illness outcomes in patients with IBD should use an illness-specific coping questionnaire, i.e., the newly developed IBD-Cope [35].

\section{Conclusions}

Despite these limitations, the present study contributes to a better understanding of the interplay between illness perceptions, coping, and adjustment to IBD. Illness perceptions and coping were shown in this cross-sectional study to influence quality of life and activity impairment. Adjustment to IBD can be improved, apart from medical interventions, by psychological interventions [34, 36-39]. The healthcare team has to anticipate on the patients disease behavior by improving the understanding of IBD and/or modifying illness perceptions and coping strategies. Briefer questionnaires with easy scoring 
schemes need to be developed to assess illness perceptions and coping strategies to improve the illness outcomes by psychological interventions too. The newly developed IBD-Cope [35] is in this way an improvement. Previous studies determined that changing illness perceptions and coping strategies is associated with improved outcomes. Chilcot et al. [36] showed in patients with irritable bowel syndrome (IBS) that illness perceptions became more positive following cognitivebehavioral therapy (CBT) compared with usual care [36]. CBT enhanced perceived control, facilitated more coherence, reduced perceptions of severe consequences, and predicted improved work and social adjustment [36]. Previous studies in IBD patients [36-39] found these effects of CBT as well, suggesting that influencing cognitive factors and behavioral aspects could lead to improved functioning in IBD. In addition, Petrie et al. [39] designed a program to modify asthma patients' illness perceptions by sending text messages that were created to push an illness perception in a direction more consistent with higher adherence [39]. In IBD patients, a text message program might be effective to increase coherent understanding of the disease and to reduce the perception of serious consequences. Based on findings of the current study, behavioral interventions increasing illness coherence, modifying perceptions of the severity of consequences and reducing the use of the maladaptive coping strategy decreasing activity may result in an increase in mental and physical health as well as less activity and work impairment.

For a health professional, it is important to pay attention to the illness perceptions of IBD patients, because they may lead to a low QoL and impairment. Knowledge and understanding of illness perceptions, coping strategies, and the effect on illness outcomes can help health professionals to understand the IBD patients' disease behaviors and improve the QoL by supporting the ability to cope with stressors related to disease. Assessing illness perceptions and coping strategies in IBD, therefore, is part and parcel of quality health care.

\section{Compliance with Ethical Standards}

Funding This study was funded by Abbott (Grant number HUM20110279).

Conflict of Interest The authors declare that they have no conflict of interest.

Ethical Approval All procedures performed in studies involving human participants were in accordance with the ethical standards of the institutional and/or national research committee and with the 1964 Helsinki Declaration and its later amendments or comparable ethical standards.

Informed Consent Informed consent was obtained from all individual participants included in the study.

\section{References}

1. Olivieri I, Cantini F, Castiglione F, Chionchetti P, Orlando A, Salvarani C, Vecchi M, Armuzzi A. Italian expert panel on the management of patients with coexisting spondyloarthritis and inflammatory bowel disease. Autoimmun Rev. 2014;13:822-30.

2. De Vlam K, Mielants H, Cuvelier C, de Keyser F, Veys EM, de Vos M. Spondyloarthropathy is underestimated in inflammatory bowel disease: prevalence and HLA association. J Rheumatol. 2000;27: 2860-5.

3. Van der Have M, Minderhoud IM, Kaptein AA, Leenders M, Siersema PD, Fidder HH, Oldenburg B. Substantial impact of illness perceptions on quality of life in patients with Crohn's disease. J Crohns Colitis. 2012;7:292-301.

4. Fuller-Thomson E, Lateef R, Sulman J. Robust association between inflammatory bowel disease and generalized anxiety disorder: findings from a nationally representative Canadian study. Inflamm Bowel Dis. 2015. doi:10.1097/MIB.0000000000000518.

5. Parekh NK, McMaster K, Nguyen DL, Shah S, Speziale A, Miller J, Melmed G. Coping strategies used by adult patients with inflammatory bowel disease. South Med J. 2015;108:337-42.

6. Casati J, Toner BB. Psychosocial aspects of inflammatory bowel disease. Biomed Pharmacother. 2000;54:388-93.

7. Casati J, Toner BB, de Rooy EC, Drossmann DA, Maunder RG. Concerns of patients with inflammatory bowel disease. Dig Dis Sci. 2000;45:26-31.

8. Bernklev T, Jahnsen J, Henriksen M, Lygren I, Aadland E, Sauar J, Schulz T, Stray N, Vatn M, Moum B. Relationship between sick leave, unemployment, disability, and health-related quality of life in patients with inflammatory bowel disease. Inflamm Bowel Dis. 2006;12:402-12.

9. Feagan BG, Bala M, Yan S, Olson A, Hanauer S. Unemployment and disability in patients with moderately to severely active Crohn's disease. J Clin Gastroenterol. 2005;39:390-5.

10. Ramos A, Calvet X, Sicilia B, Vergara M, Figuerola A, Motos J, Sastre A, Villoria A, Gomollon F. IBD-related work disability in the community: prevalence, severity and predictive factors. A crosssectional study. United European Gastroenterol J. 2015;3(4):33542.

11. Leventhal H, Brissette I, Leventhal EA. The Common-Sense Model of self-regulation of health and illness. In: Cameron LD, Leventhal $\mathrm{H}$, editors. The self-regulation of health and illness behaviour, vol. 2003. London: Routledge; 2003. p. 42-65.

12. Dorrian A, Dempster M, Adair P. Adjustment to inflammatory bowel disease: the relative influence of illness perceptions and coping. Inflamm Bowel Dis. 2009;15:47-55.

13. Kiebles JL, Doerfler B, Keefer L. Preliminary evidence supporting a framework of psychological adjustment to inflammatory bowel disease. Inflamm Bowel Dis. 2010;16:1685-95.

14. Knowles SR, Cook SI, Tribbick D. Relationship between health status, illness perceptions, coping strategies and psychological morbidity: a preliminary study with IBD stoma patients. J Crohns Colitis. 2013;7:471-8.

15. Knowles SR, Grass C, Macrae F. Illness perceptions in IBD influence psychological status, sexual health and satisfaction, body image and relational functioning: a preliminary exploration using structural equation modeling. J Crohns Colitis. 2013;7:344-50.

16. Knowles SR, Wilson JL, Connell WR, Kamm MA. Preliminary examination of the relations between disease activity, illness perceptions, coping strategies, and psychological morbidity in Crohn's disease guided by the Common Sense Model of illness. Inflamm Bowel Dis. 2011;17:2551-7.

17. Rochelle TL, Fidler H. The importance of illness perceptions, quality of life and psychological status in patients with ulcerative colitis and Crohn's disease. J Health Psychol. 2012;18:972-83. 
18. Moss-Morris R, Weinman J, Petrie KJ, Horne R, Cameron LD, Buick D. The Revised Illness Perception Questionnaire (IPQ-R). Psychol Health Med. 2002;17:1-16.

19. Van Lankveld W, van't Pad Bosch P, van de Putte L, Näring G, van der Staak C. Disease-specific stressors in rheumatoid arthritis: coping and well-being. Br J Rheumatol. 1994;33:1067-73.

20. Haggar SM, Orbell S. A meta-analytic review of the CommonSense Model of illness representations. Psychol Health. 2003;18(2):141-84.

21. Han SW, McColl E, Barton JR, James P, Steen IN, Walfare MR. Predictors of quality of life in ulcerative colitis. The importance of symptoms and illness representations. Inflamm Bowel Dis. 2005;11:24-34.

22. Van der Have M, Brakenhoff LKPM, van Erp SJH, Kaptein AA, Leenders M, Scharloo M, Veenendaal RA, van der Heijde D, van der Meulen-de Jong AE, Hommes DW, Fidder HH. Back/joint pain, illness perceptions and coping are important predictors of quality of life and work productivity in patients with inflammatory bowel disease: a 12-month longitudinal study. J Crohns Colitis. 2015;9:276-83.

23. Van der Have M, Fidder HH, Leenders M, Kaptein AA, van der Valk ME, van Bodegraven AA, Dijkstra G, de Jong DJ, Pierik M, Ponsioen CY, van der Meulen-de Jong AE, van der Woude CJ, van de Meeberg PC, Romberg-Camps MJ, Clemens CH, Jansen JM, Mahmmod N, Bolwerk CJ, Vermeijden JR, Siersema PD, Oldenburg B. Self-reported disability in patients with inflammatory bowel disease largely determined by disease activity and illness perceptions. Inflamm Bowel Dis. 2015;21:369-77.

24. Netjes JE, Rijken M. Labor participation among IBD patients with inflammatory bowel disease. Inflamm Bowel Dis. 2013;19(1):8191.

25. McCombie AM, Mulder RT, Gearry RB. Coping strategies and psychological outcomes of patients with inflammatory bowel disease in the first 6 months after diagnosis. Inflamm Bowel Dis. 2015;21(10):2272-80.

26. Van Erp SJH, Brakenhoff LK, van Gaalen FA, van den Berg R, Fidder HH, Verspaget HW, Huizinga TW, Veenendaal RA, Wolterbeek R, van der Heijde D, van der Meulen-de Jong AE, Hommes DW. Classifying back pain and peripheral joint complaints in inflammatory bowel disease patients: a prospective longitudinal follow-up study. J Crohns Colitis. 2015;9(3):276-83. doi:10.1093/ecco-jcc/jju025.

27. Harvey RF, Bradshaw JMA. Simple index of Crohn's disease activity. Lancet. 1980;1(8173):876.
28. Walmsley RS, Ayres RC, Pounder RE, Allan RNA. Simple clinical colitis activity index. Gut. 1998;43:29-32.

29. Ware JE, Sherbourne CD. The MOS 36-item short-form health survey (SF-36). I. Conceptual framework and item selection. Med Care. 1992;30:473-83.

30. Zhang W, Bansback N, Boonen A, Young A, Singh A, Anis AH. Validity of the work productivity and activity impairment questionnaire - general health version in patients with rheumatoid arthritis. Arthritis Res Ther. 2010. doi:10.1186/ar3141.

31. Reilly MC, Gerlier L, Brabant Y, Brown M. Validity, reliability, and responsiveness of the work productivity and activity impairment questionnaire in Crohn's disease. Clin Ther. 2008;30:393-404.

32. Hayes AF. Introduction to mediation, moderation, and conditional process analysis: a regression-based approach. New York: Guilford Press; 2013.

33. Hayes AF, Preacher KJ. Statistical mediation analysis with a multicategorical independent variable. Br J Math Stat Psychol. 2014;67:451-70.

34. Tiggelman D, van de Ven MOM, van Schayck OCP, Kleinjan M, Engels RCME. The Common Sense Model in early adolescents with asthma: longitudinal relations between illness perceptions, asthma control and emotional problems mediated by coping. J Psychosom Res. 2014;77(4):309-15.

35. McCombie A, Swaminathan A, Mulder R, Frampton C, Kortlever T, Gearry R. The IBD-Cope: a new instrument for measuring coping in inflammatory bowel disease patients. J Crohns Colitis. 2016;10(6):678-86.

36. Chilcot H, Moss-Morris R. Changes in illness-related cognitions rather than distress mediate improvements in irritable bowel syndrome (IBS) symptoms and disability following a brief cognitive behavioural therapy intervention. Behav Res Ther. 2013;51:690-5.

37. Mikocka-Walus A, Bampton P, Hetzel D, Hughes D, Esterman A, Andrews JM. Cognitive-behavioural therapy has no effect on disease activity but improves quality of life in subgroups of patients with inflammatory bowel disease: a pilot randomized controlled trial. BMC Gastroenterol. 2015. doi:10.1186/s12876-015-0278-2.

38. Mussell M, Bocker U, Nagel N, Olbrich R, Singer MV. Reducing psychological distress in patients with inflammatory bowel disease by cognitive-behavioural treatment: exploratory study of effectiveness. Scand J Gasteroenterol. 2003;38(7):755-62.

39. Petrie KJ, Perry K, Broadbent E, Weinman J. A text message programme designed to modify patients' illness and treatment beliefs improves self-reported adherence to asthma preventer medication. Br J Health Psychol. 2012;17:74-84. 